\title{
Coccidae, Pseudococcidae, Ortheziidae, and Monophlebidae (Hemiptera: Coccoidea) of Espírito Santo, Brazil
}

\author{
Mark Paul Culik ${ }^{1,4}$, David dos Santos Martins ${ }^{1}$, José Aires Ventura ${ }^{1}$, \\ Ana Lúcia Benfatti Gonzalez, Peronti², Penny Jean Gullan³, \& Takumasa Kondo ${ }^{3}$ \\ Biota Neotropica v7 (n3) - http://www.biotaneotropica.org.br/v7n3/pt/abstract?article +bn00507032007 \\ Data Received 04/05/07 \\ Revised 24/07/07 \\ Accepted 01/09/07 \\ ${ }^{1}$ Instituto Capixaba de Pesquisa, Assistência Técnica e Extensão Rural-INCAPER, \\ Rua Afonso Sarlo, 160, \\ CEP 29052-010, Vitória, ES, Brasil \\ ${ }^{2}$ Departamento de Ecologia e Biologia Evolutiva, Universidade Federal de São Carlos - UFSCar, \\ CP 676, CEP 13565-905, São Carlos, SP, Brasil \\ ${ }^{3}$ Department of Entomology, University of California, Davis, \\ One Shields Avenue, Davis, CA 95616-8584, USA \\ ${ }^{4}$ Corresponding author: Mark Paul Culik, e-mail: markculik@hotmail.com
}

\begin{abstract}
Culik, M.P., Martins, D.S., Ventura, J.A., Peronti, A.L.B.G., Gullan, P.Y. \& Kondo, T. Coccidae, Pseudococcidae, Ortheziidae, and Monophlebidae (Hemiptera: Coccoidea) of Espírito Santo, Brazil. Biota Neotrop. Sep/Dez 2007 vol. 7, no. 3 http://www.biotaneotropica.org.br/v7n3/pt/abstract?article+bn00507032007. ISSN 1676-0603.

New plant hosts are recorded for nine scale insect species recently collected in Espírito Santo, Brazil, and eleven scale insect species are recorded for the first time from the state: Ceroplastes floridensis Comstock, Coccus longulus (Douglas), Coccus viridis (Green), Eucalymnatus tesselatus (Signoret), Pseudokermes sp., Saissetia coffeae (Walker), Phenacoccus madeirensis Green, Pseudococcus jackbeardsleyi Gimpel \& Miller, Pseudococcus longispinus (Targioni Tozzetti), Icerya purchasi Maskell, and Icerya genistae Hempel. This is also the first record of Co. longulus in Brazil. Information on the host plants and geographic distribution of the 26 species of scale insects of the families Coccidae, Pseudococcidae, Ortheziidae, and Monophlebidae, currently known from Espírito Santo is provided.
\end{abstract}

Keywords: scale insects, invasive species, Icerya genistae, biodiversity, biogeography.

\section{Resumo}

Culik, M.P., Martins, D.S., Ventura, J.A., Peronti, A.L.B.G., Gullan, P.Y. \& Kondo, T. Coccidae, Pseudococcidae, Ortheziidae, e Monophlebidae (Hemiptera: Coccoidea) do Espírito Santo, Brasil. Biota Neotrop. Sep/Dez 2007 vol. 7, no. 3 http://www.biotaneotropica.org.br/v7n3/pt/abstract?article+bn00507032007. ISSN 1676-0603.

Novas plantas hospedeiras foram registradas para nove espécies de cochonilhas coletadas recentemente no Estado do Espírito Santo, Brasil, e onze espécies de cochonilhas são registradas pela primeira vez no Estado: Ceroplastes floridensis Comstock, Coccus longulus (Douglas), Coccus viridis (Green), Eucalymnatus tesselatus (Signoret), Pseudokermes sp., Saissetia coffeae (Walker), Phenacoccus madeirensis Green, Pseudococcus jackbeardsleyi Gimpel \& Miller, Pseudococcus longispinus (Targioni Tozzetti), Icerya purchasi Maskell, e Icerya genistae Hempel. Co. longulus é registrada pela primeira vez no Brasil. É disponibilizada a informação das plantas hospedeiras e a distribuição geográfica de 26 espécies de cochonilhas das famílias Coccidae, Pseudococcidae, Ortheziidae, e Monophlebidae atualmente conhecidas no Estado do Espírito Santo.

Palavras-chave: cochonilhas, espécies invasoras, Icerya genistae, biodiversidade, biogeografia. 


\section{Introduction}

Scale insects (Hemiptera: Coccoidea) are sap feeding pests of many agricultural crops and ornamental plants (Miller et al. 2005) but relatively little is known of the scale insect fauna of Espírito Santo, Brazil (Silva et al. 1968, Ben-Dov et al. 2006). Scale insects are difficult to identify and it is likely that the lack of knowledge of scale insects in this area is because there are few people in South America with the taxonomic expertise to identify these insects. Accurate identifications of scale insects requires the preparation of high quality microscope slide-mounts; careful examination of specimens using a compound microscope and high magnification; familiarity with a relatively great variety of morphological structures unique to this group of insects; and access to the widely dispersed taxonomic literature, and especially keys, essential for such identifications. Lack of knowledge of scale insects in this area may also be because these insects have been generally unnoticed (and of little interest) because natural enemies have been effective in maintaining populations at low levels, or they have been tolerated because control has not been economically justified. And, there is a danger that current changes in agriculture in Brazil (Alves 2003), and similar areas, may lead to greater problems caused by scale insects due to destruction of natural enemies or other factors associated with such changes. The objective of our studies of scale insects in Espírito Santo (Martins et al. 2004, Culik \& Gullan 2005, Kondo et al. 2005, Culik et al. 2006) is to address the lack of knowledge of these insects in this area and specifically, this study was conducted as part of our efforts to identify the scale insect pests of papaya, pineapple and other economically important plants in this state.

\section{Materials and Methods}

Scale insects (Coccidae, Pseudococcidae, Ortheziidae, and Monophlebidae) were collected during surveys of the insect fauna of papaya and pineapple and when noticed on plants during field work or other activities in Espírito Santo during 2004 to 2006. Samples of plants or plant parts (fruits, leaves, stems) infested with scale insects were collected from various locations in the state ranging from municipalities of Pedro Canário in the north $\left(18^{\circ} 17^{\prime} 24^{\prime \prime} \mathrm{S}\right.$ and $\left.39^{\circ} 57^{\prime} 36^{\prime \prime} \mathrm{W}\right)$ to Marataízes in the south $\left(21^{\circ} 01^{\prime} 48^{\prime \prime} \mathrm{S}\right.$ and $\left.40^{\circ} 49^{\prime} 48^{\prime \prime} \mathrm{W}\right)$ and Vitória on the coast $\left(20^{\circ} 19^{\prime} 12^{\prime \prime} \mathrm{S}\right.$ and $40^{\circ} 21^{\prime} 00^{\prime}$ ' W) to Cachoeiro de Itapemirim $\left(20^{\circ} 49^{\prime} 48^{\prime \prime} \mathrm{S}\right.$ and $\left.41^{\circ} 11^{\prime} 24^{\prime \prime} \mathrm{W}\right)$ in the interior of the state. The samples were transported to the Espírito Santo research and rural extension institute INCAPER (Instituto Capixaba de Pesquisa, Assistência Técnica e Extensão Rural) headquarters in Vitória for photographing and preservation of the scale insects and representative specimens were sent to taxonomic specialists for confirmation of identifications. Taxonomists responsible for confirming the identifications of the insects were T. Kondo: Coccidae, Pseudococcidae; A. Peronti: Coccidae, Pseudococcidae, Ortheziidae; G. Evans: Pseudococcidae; M. Kaydan: Pseudococcidae; P. Gullan: Ortheziidae, Monophlebidae; and C.Unruh: Monophlebidae. Voucher specimens of the insects collected are deposited in the arthropod collections of INCAPER, Vitória, Espírito Santo, the Bohart Museum of Entomology, University of California, Davis, and the Coleção Entomológica do Departamento de Ecologia e Biologia Evolutiva, Universidade Federal de São Carlos - UFSCar, São Paulo.

\section{Results and Discussion}

Eighteen scale insect species were newly identified from Espírito Santo in this study of which eleven are recorded here for the first time from the state: Ceroplastes floridensis Comstock,
Coccus longulus (Douglas), Coccus viridis (Green), Eucalymnatus tesselatus (Signoret), Pseudokermes sp., Saissetia coffeae (Walker), Phenacoccus madeirensis Green, Pseudococcus jackbeardsleyi Gimpel \& Miller, Pseudococcus longispinus (Targioni Tozzetti), Icerya purchasi Maskell, and Icerya genistae Hempel (Table 1). These records almost double the total number of scale insects of the families Coccidae, Pseudococcidae, Ortheziidae, and Monophlebidae known from this state to 26 species. This is also the first record of C. longulus in Brazil, which is of significance because this species, probably of Oriental origin (Miller et al. 2005), is a potential pest of many economically important crops (Ben-Dov 2006a).

Although most of these scale insects are known to be polyphagous (Ben-Dov 2006a, Ben-Dov 2006b, Ben-Dov 2006c, Miller \& Gimpel 2006), results of this study are also of note because new host plants are recorded for several of the species, including Phenacoccus solenopsis Tinsley, recorded here for the first time from the host plant families Amaranthaceae and Caricaceae. Ph. solenopsis is very common in Espírito Santo and has been previously noted here as a pest of tomato (Culik \& Gullan 2005).

In addition, these are the first records of Planococcus citri (Risso) and Ps. jackbeardsleyi from Coffea canephora, and Dysmicoccus brevipes (Cockerell) was also identified from rosettes of this economically important crop. In Espírito Santo mealybugs that infest the inflorescences of coffee plants are major pests of coffee (Fornazier 2006) commonly referred to simply as the "cochonilha da roseta" (rosette mealybug) but information regarding the species that actually occur on coffee in this region is lacking (Santa-Cecília et al. 2002). Results of this study provide additional evidence that in fact a complex of mealybug species may attack inflorescences of C. canephora and indicate the need for further research to determine the extent to which these various mealybug species contribute to reductions in coffee yields in Espírito Santo so these insects can be managed with greater effectiveness.

The monophlebid species Icerya genistae was described from Brazil in 1912 (Hempel 1912) but remained relatively unnoticed until recently when it was found in the Caribbean and Florida (Ben-Dov 2006b, Hodges 2006). The species is polyphagous and has been noted to be a serious pest of vegetable crops in Barbados (Hodges 2006). Thus, additional information on the species, such as its natural enemies in its area of origin, may be needed for management of this pest in the future.

Accurate knowledge of the insects present in an area is essential as a basis for development of integrated pest management. Thus, the information on scale insects and their associated host plants in Espírito Santo obtained in the present study should enable researchers and producers to more effectively develop and utilize integrated pest management methods for production of crops in this state. Results of this study also confirm that a diverse variety of scale insect species are present in Espírito Santo and indicate the need for researchers and producers to develop and utilize integrated pest management methods to avoid practices that may favor the development of these potential pests in the future. Information obtained in this study is also likely to be of interest in other regions in unfortunate instances of the introduction of pest species into new areas (as appears to have happened with I. genistae), and as a contribution to a more complete understanding of the host plants and geographical distribution of scale insects in general.

\section{Acknowledgments}

We thank C. M. Unruh, University of California, Davis, G. A. Evans, USDA, Beltsville, and M. B. Kaydan, Yüzüncü Yil University, Turkey, for identifying some of the scale insects collected 
Table 1. Coccidae, Pseudococcidae, Ortheziidae, and Monophlebidae of Espírito Santo (ES), Brazil: this study (2004 - 2006) and previous records.

Tabela 1. Coccidae, Pseudococcidae, Ortheziidae e Monophlebidae do Espírito Santo (ES), Brasil.

\begin{tabular}{|c|c|c|c|c|}
\hline Species, common name & $\begin{array}{c}\text { Collection } \\
\text { municipality; No. }\end{array}$ & $\begin{array}{c}\text { Host plants in ES previous / } \\
\text { present study }\end{array}$ & $\begin{array}{l}\text { Geographic } \\
\text { distribution }\end{array}$ & $\begin{array}{l}\text { Reference, } \\
\text { Note }^{2}\end{array}$ \\
\hline \multicolumn{5}{|l|}{ COCCIDAE } \\
\hline $\begin{array}{l}\text { Ceroplastes floridensis } \\
\text { Comstock, Florida wax scale }\end{array}$ & Linhares & $\begin{array}{l}\text { Coffea canephora } \\
\text { (inflorescence) }\end{array}$ & Cosmopolitan & - \\
\hline $\begin{array}{l}\text { Coccus hesperidum Linnaeus, } \\
\text { brown soft scale }\end{array}$ & $\begin{array}{l}\text { Linhares, Sooretama, } \\
\text { Vitória; } 16\end{array}$ & $\begin{array}{l}\text { Carica papaya }(\text { stem }) / \\
\text { Carica papaya, Dietes bicolor, } \\
\text { Solanum americanum }\end{array}$ & Cosmopolitan & $\begin{array}{l}\text { Martins et al. } \\
2004 \\
\text { New host: } \\
\text { Solanum } \\
\text { americanum }\end{array}$ \\
\hline $\begin{array}{l}\text { Coccus longulus (Douglas), } \\
\text { long brown scale }\end{array}$ & Vitória & Spathiphyllum wallisi & Cosmopolitan & $\begin{array}{l}\text { New host: } \\
\text { Spathiphyllum } \\
\text { wallisi }\end{array}$ \\
\hline $\begin{array}{l}\text { Coccus viridis (Green), } \\
\text { green scale }\end{array}$ & $\begin{array}{l}\text { Aracruz, Linhares, } \\
\text { Vitória; } 3\end{array}$ & $\begin{array}{l}\text { Coffea canephora } \\
\text { (inflorescence, stem), Murraya } \\
\text { paniculata }\end{array}$ & Cosmopolitan & - \\
\hline $\begin{array}{l}\text { Eucalymnatus tesselatus } \\
\text { (Signoret) }\end{array}$ & Vitória & Dypsis lutescens & Cosmopolitan & - \\
\hline Pseudokermes sp. & Vitória & unidentified plant & & - \\
\hline $\begin{array}{l}\text { Saissetia coffeae (Walker), } \\
\text { hemispherical scale }\end{array}$ & $\begin{array}{l}\text { Domingos Martins, } \\
\text { Linhares, } \\
\text { Sooretama; } 6\end{array}$ & $\begin{array}{l}\text { Citrus sp., Coffea canephora } \\
\text { (inflorescence, trunk), Bidens } \\
\text { pilosa (root) }\end{array}$ & Cosmopolitan & $\begin{array}{l}\text { New host: } \\
\text { Bidens }\end{array}$ \\
\hline \multicolumn{5}{|l|}{ PSEUDOCOCCIDAE } \\
\hline $\begin{array}{l}\text { Antonina graminis (Maskell), } \\
\text { Rhodesgrass mealybug }\end{array}$ & Serra & Cynodon dactylon & Cosmopolitan & $\begin{array}{l}\text { Culik \& Gullan } \\
2005\end{array}$ \\
\hline $\begin{array}{l}\text { Dysmicoccus boninsis (Kuwana), } \\
\text { gray sugarcane mealybug }\end{array}$ & Serra & Saccharum officinarum & Widespread & $\begin{array}{l}\text { Culik \& Gullan } \\
2005\end{array}$ \\
\hline $\begin{array}{l}\text { Dysmicoccus brevipes } \\
\text { (Cockerell), pineapple mealybug }\end{array}$ & $\begin{array}{l}\text { Aracruz, Cachoeiro } \\
\text { de Itapemirim, João } \\
\text { Neiva, Linhares, } \\
\text { Marataízes, Pedro } \\
\text { Canário, Pinheiros, } \\
\text { Serra, Sooretama, } \\
\text { Vitória; } 24\end{array}$ & $\begin{array}{l}\text { Ananas comosus, Cucurbita } \\
\text { pepo / A. comosus, Coffea } \\
\text { canephora (inflorescence), } \\
\text { Psidium guajava (root) }\end{array}$ & Cosmopolitan & $\begin{array}{l}\text { Culik \& Gullan } \\
2005\end{array}$ \\
\hline Dysmicoccus grassii (Leonardi) & Aracruz, Linhares & $\begin{array}{l}\text { Carica papaya (fruit), Coffea } \\
\text { canephora (inflorescence) / } \\
\text { Carica papaya }\end{array}$ & $\begin{array}{l}\text { Neotropical and } \\
\text { few other areas }\end{array}$ & Culik et al. 2006 \\
\hline Ferrisia malvastra (McDaniel) & Linhares & Bidens pilosa (roots) & Widespread & Culik et al. 2006 \\
\hline $\begin{array}{l}\text { Ferrisia virgata (Cockerell), } \\
\text { striped mealybug }\end{array}$ & $\begin{array}{l}\text { Linhares, Serra, } \\
\text { Vitória; } 3\end{array}$ & $\begin{array}{l}\text { weed cf. Spermacoce sp. } \\
\text { (leaf, stem) / Citrus sp. } \\
\text { (stem), Ranunculus repens, } \\
\text { unidentified plant (root) }\end{array}$ & Cosmopolitan & $\begin{array}{l}\text { Culik et al. } \\
2006 ; \\
\text { New host: } \\
\text { Ranunculaceae }\end{array}$ \\
\hline Phenacoccus sp. & $\begin{array}{l}\text { Linhares, } \\
\text { Sooretama; } 5\end{array}$ & $\begin{array}{l}\text { Bidens pilosa (root), } \\
\text { unidentified plant (root) }\end{array}$ & - & - \\
\hline $\begin{array}{l}\text { Phenacoccus madeirensis } \\
\text { Green, madeira mealybug }\end{array}$ & Serra & $\begin{array}{l}\text { weed cf. Spermacoce sp. } \\
\text { (leaf/stem) }\end{array}$ & Cosmopolitan & - \\
\hline $\begin{array}{l}\text { Phenacoccus solenopsis } \\
\text { Tinsley }\end{array}$ & $\begin{array}{l}\text { Linhares, Serra, } \\
\text { Vitória; } 4\end{array}$ & $\begin{array}{l}\text { Solanum lycopersicum, weed } \\
\text { cf. Spermacoce sp. I } \\
\text { Amaranthus flavus (stem), } \\
\text { Bidens pilosa, (stem), Carica } \\
\text { papaya (trunk, fruit), Emilia } \\
\text { sonchifolia (root) }\end{array}$ & $\begin{array}{l}\text { Nearctic, } \\
\text { Neotropical }\end{array}$ & $\begin{array}{l}\text { Culik \& Gullan } \\
\text { 2005; New hosts: } \\
\text { Amaranthaceae, } \\
\text { Bidens, } \\
\text { Caricaceae }\end{array}$ \\
\hline
\end{tabular}


Table 1. Continued...

\begin{tabular}{|c|c|c|c|c|}
\hline Species, common name & $\begin{array}{c}\text { Collection } \\
\text { municipality; No. }{ }^{1}\end{array}$ & $\begin{array}{c}\text { Host plants in ES previous / } \\
\text { present study }\end{array}$ & $\begin{array}{l}\text { Geographic } \\
\text { distribution }\end{array}$ & $\begin{array}{l}\text { Reference, } \\
\text { Note }^{2}\end{array}$ \\
\hline $\begin{array}{l}\text { Phenacoccus tucumanus } \\
\text { Granara de Willink }\end{array}$ & Serra & Citrus sp. (leaf) & Neotropical & Culik et al. 2006 \\
\hline $\begin{array}{l}\text { Planococcus citri (Risso), } \\
\text { citrus mealybug }\end{array}$ & $\begin{array}{l}\text { Aracruz, Linhares, } \\
\text { Sooretama, Viana, } \\
\text { Vitória; } 23\end{array}$ & $\begin{array}{l}\text { Bidens pilosa (root), Citrus } \\
\text { sp. (fruit), Coffea canephora } \\
\text { (inflorescence, root, trunk, leaf), } \\
\text { Leea rubra, Lepidium virginicum } \\
\text { (root), unidentified plant }\end{array}$ & Cosmopolitan & $\begin{array}{l}\text { Silva et al. 1968; } \\
\text { New hosts: } \\
\text { Bidens, Coffea } \\
\text { canephora, } \\
\text { Leea, Lepidium }\end{array}$ \\
\hline Planococcus minor (Maskell) & $\begin{array}{l}\text { Castelo, Linhares, } \\
\text { Sooretama }\end{array}$ & $\begin{array}{l}\text { Bidens pilosa (roots), Coffea sp.; } \\
\text { Coffea canephora } \\
\text { (inflorescence) }\end{array}$ & Widespread & $\begin{array}{l}\text { Santa-Cecília } \\
\text { et al. } 2002, \\
\text { Culik et al. } 2006\end{array}$ \\
\hline Plotococcus capixaba Kondo & $\begin{array}{l}\text { Alfredo Chaves, } \\
\text { Serra }\end{array}$ & $\begin{array}{l}\text { Eugenia } \text { cf. pitanga (leaf), } \\
\text { Myrciaria jaboticaba (leaf) }\end{array}$ & $\begin{array}{l}\text { Brazil: ES, } \\
\text { São Paulo }\end{array}$ & $\begin{array}{l}\text { Kondo et al. } \\
\text { 2005, Culik } \\
\text { et al. } 2006\end{array}$ \\
\hline Pseudococcus sp. & $\begin{array}{l}\text { Linhares, } \\
\text { Sooretama; } 2\end{array}$ & $\begin{array}{l}\text { Coffea canephora (root), Carica } \\
\text { papaya (stem) }\end{array}$ & - & - \\
\hline $\begin{array}{l}\text { Pseudococcus elisae } \\
\text { Borchsenius }\end{array}$ & Aracruz, Serra & $\begin{array}{l}\text { Coffea canephora (inflorescence), } \\
\text { unidentified weed }\end{array}$ & $\begin{array}{l}\text { Nearctic, } \\
\text { Neotropical }\end{array}$ & Culik et al. 2006 \\
\hline $\begin{array}{l}\text { Pseudococcus jackbeardsleyi } \\
\text { Gimpel \& Miller }\end{array}$ & $\begin{array}{l}\text { Aracruz, Cachoeiro } \\
\text { de Itapemirim, Lin- } \\
\text { hares, Marataízes, } \\
\text { Serra; } 9\end{array}$ & $\begin{array}{l}\text { Ananas comosus, } \\
\text { Coffea canephora, (inflorescence), } \\
\text { Cucurbita pepo (fruit), } \\
\text { Manihot esculenta (leaf, root), } \\
\text { weed cf. Spermacoce sp. } \\
\text { (leaf/stem) }\end{array}$ & Widespread & $\begin{array}{l}\text { New host: } \\
\text { Coffea } \\
\text { canephora, } \\
\text { Cucurbita }\end{array}$ \\
\hline $\begin{array}{l}\text { Pseudococcus longispinus } \\
\text { (Targioni Tozzetti), longtailed } \\
\text { mealybug }\end{array}$ & Vilha Velha & Dioscorea sp. & Cosmopolitan & $\begin{array}{l}\text { New host: } \\
\text { Dioscoreaceae }\end{array}$ \\
\hline $\begin{array}{l}\text { Pseudococcus viburni } \\
\text { (Signoret), obscure mealybug }\end{array}$ & Vitória & Solanum tuberosum & Cosmopolitan & $\begin{array}{l}\text { Culik \& Gullan } \\
2005\end{array}$ \\
\hline \multicolumn{5}{|l|}{ ORTHEZIIDAE } \\
\hline $\begin{array}{l}\text { Praelongorthezia praelonga } \\
\text { (Douglas) }\end{array}$ & Linhares; Vitória; 4 & $\begin{array}{l}\text { Coffea canephora (leaf), Citrus } \\
\text { sp., (leaf), Schefflera sp., } \\
\text { Tabebuia sp. (leaf) }\end{array}$ & $\begin{array}{l}\text { Neotropical } \\
\text { and Mexico }\end{array}$ & $\begin{array}{l}\text { Martins et al. } \\
\text { 1989; } \\
\text { New host: } \\
\text { Araliaceae }\end{array}$ \\
\hline \multicolumn{5}{|l|}{ MONOPHLEBIDAE } \\
\hline $\begin{array}{l}\text { Icerya purchasi Maskell, } \\
\text { cottony cushion scale }\end{array}$ & Serra & Cajanus cajan & Cosmopolitan & - \\
\hline Icerya genistae Hempel & Serra & unidentified legume & $\begin{array}{l}\text { Brazil, Carib- } \\
\text { bean, Florida }\end{array}$ & - \\
\hline
\end{tabular}

Species collected in this study (2004-2006) are listed in bold font;

${ }^{1}$ No. = number of samples is indicated if the species was identified from more than one sample in this study;

${ }^{2}$ Reference cited if previously recorded from Espírito Santo.

in this study. M. B. Kaydan also prepared a number of slide-mounts to enable species identification for this study. T. Kondo was supported by the U.S. National Science Foundation (Partnership for Enhancing Expertise in Taxonomy program, under Grant No. 0118718 to P. J. Gullan). The Conselho Nacional de Desenvolvimento Científico e Tecnológico - CNPq, Banco do Nordeste do Brasil - BNB, Fundação de Apoio à Ciência e Tecnologia do Espírito Santo - FAPES, Financiadora de Estudos e Projetos - FINEP, and Ministério da Agricultura, Pecuária e Abastecimento - MAPA, Brazil, provided financial support for this work.

\section{References}

ALVES, F. DE L. 2003. A cultura do mamão Carica papaya no mundo, no Brasil e no Estado do Espírito Santo. In A cultura do mamoeiro: tecnologias de produção (D. dos S. Martins \& A. de F.S. da Costa, eds), INCAPER, Vitória, p. 13-34.

BEN-DOV, Y. 2006a. ScaleNet, Coccidae. http://www.sel.barc.usda.gov/scalecgi/chklist.exe?Family=Coccidae \&genus= (último acesso em 12/02/07).

BEN-DOV, Y. 2006b. ScaleNet, Margarodidae. http://www.sel.barc.usda. gov/scalecgi/chklist.exe?Family=Margarodidae\&genus= (último acesso em 12/02/07).

BEN-DOV, Y. 2006c. ScaleNet, Pseudococcidae. http://www.sel.barc.usda. gov/scalecgi/chklist.exe?Family=Pseudococcidae \&genus= (último acesso em 12/02/2007).

BEN-DOV, Y., MILLER, D.R. \& GIBSON, G.A.P. 2006. ScaleNet, Scales in a country query results. http://www.sel.barc.usda.gov/scalecgi/region. exe?region $=\mathrm{T} \&$ family $=$ All $\&$ country $=$ BRA $\&$ genus $=\&$ subunit $=$ ES \&intro $=$ A\&detail $=$ Yes\&regname $=$ Neotropical $\&$ ctryname $=$ Brazil\&action $=$ Sub mit+Query\&querytype=Subunit+Query (último acesso em 12/02/07). 
CULIK, M.P. \& GULLAN, P.J. 2005. A new pest of tomato and other records of mealybugs (Hemiptera: Pseudococcidae) from Espírito Santo, Brazil. Zootaxa 964:1-8.

CULIK, M.P., MARTINS, D.S. \& P.J. GULLAN, P.J. 2006. First records of two mealybug species in Brazil and new potential pests of papaya and coffee. J. Insect Sci. 6(23):1-6.

FORNAZIER, M.J. 2006. Eficiência agronômica do uso de Lorsban 480 BR no controle da cochonilha da roseta, Planococcus minor. http:// www.dowagro.com/PublishedLiterature/dh_05ae/09002f13805aebf0. pdf?filepath=br/pdfs/noreg/013-02056.pdf\&fromPage=GetDoc (último acesso em 16/02/07).

HEMPEL, A. 1912. As coccidas do Brasil. Catálogo da fauna Brasileira. Ed. Mus. Paul., São Paulo.

HODGES, G. 2006. Icerya genistae (Hemiptera: Margarodidae) a new exotic scale insect for Florida. http://www.doacs.state.fl.us/pi/enpp/ento/ i.genistae.html (último acesso em 12/02/07).

KONDO, T., GULLAN, P.J., VENTURA, J.A. \& CULIK, M.P. 2005. Taxonomy and biology of the mealybug genus Plotococcus Miller \& Denno (Hemiptera: Pseudococcidae) in Brazil, with descriptions of two new species. Stud. Neotrop. Fauna E. 40:213-227.
MARTINS, D.S., PAULINI, A.E. \& GALVÃO, M.M. 1989. Incidência de Orthezia praelonga Douglas 1891, em café conilon no Estado do Espírito Santo. Articulação Pesquisa-Extensão, 9. EMCAPA, Vitória.

MARTINS, D. DOS S., CULIK, M.P. \& WOLFF, V.R. DOS S. 2004. New record of scale insects (Hemiptera: Coccoidea) as pests of papaya in Brazil. Neotrop. Entomol. 33:655-657.

MILLER, D.R. \& GIMPEL, M.E. 2006. ScaleNet, Ortheziidae. Available from http://www.sel.barc.usda.gov/scalecgi/chklist.exe?Family=Orthez iidae \&genus $=($ accessed 12 February 2007)

MILLER, D.R., MILLER, G.L., HODGES, G.S. \& DAVIDSON, J.A. 2005. Introduced scale insects (Hemiptera: Coccoidea) of the United States and their impact on U.S. agriculture. P. Entomol. Soc. Wash. 107:123-158.

SANTA-CECÍLIA, L.V.C., REIS, P.R. \& SOUZA, J.C. 2002. Sobre a nomenclatura das espécies de cochonilhas-farinhentas do cafeeiro nos Estados de Minas Gerais e Espírito Santo. Neotrop. Entomol. 31:333-334.

SILVA, A.G.A., GONÇALVES, C.R., GALVÃO, D.M., GONÇALVES, A.J.L., GOMES, J., SILVA, M. DO N. \& SIMONI, L. DE. 1968. Quarto catálogo dos insetos que vivem nas plantas do Brasil seus parasitos e predadores. Parte II $-1^{\circ}$ Tomo. Ministério da Agricultura, Rio de Janeiro. 
